\title{
Equation of conservation of irradiance
}

Carlos Robledo-Sanchez, A. Jaramillo, D. Gale, Gilberto Camacho

Carlos I. Robledo-Sanchez, A. Jaramillo, D. M. Gale, Gilberto Camacho, "Equation of conservation of irradiance," Proc. SPIE 3831, Sixth International Conference on Education and Training in Optics and Photonics, (16 June 2000); doi: $10.1117 / 12.388741$

Event: Education and Training in Optics and Photonics (ETOP'99), 1999, Cancun, Mexico 


\title{
Equation of conservation of irradiance
}

\author{
C.I: Robledo-Sánchez, A. Jaramillo*, D. Gale*, G. Camacho \\ FCFM, BUAP, Apdo. Postal 1152, Puebla, Pue. México, 72000 \\ *INAOE, Apdo. Postal 51 and 216, Puebla, Pue. , México. 72000 \\ E-mail: crobledo@fcfm.buap.mx, ajaramil@inaoep.mx.
}

\begin{abstract}
The equation for the conservation of irradiance is deduced from the paraxial wave equation. This formulation is presented in an optics Masters course, with the aim of showing to students its utility in the recuperation of the phase of a wavefront with just the measurement of irradiance.
\end{abstract}

Key words: Paraxial equation, aberration-function, law of conservation.

\section{THEORY}

From the paraxial wave equation

$$
\left(\nabla^{2}+2 i k \frac{\partial}{\partial z}\right) u=0
$$

where $\nabla^{2}$ is the Laplacian operator $\left(\frac{\partial^{2}}{\partial x^{2}}+\frac{\partial^{2}}{\partial y^{2}}\right)$, and $u(r)=I^{1 / 2}(r) \exp (i \varphi(r))$. Multiplying the left side of equation (1) by $u^{*}(r)$ we obtain

$$
2 i k u * \frac{\partial}{\partial z} u+u * \nabla^{2} u=0
$$

Now taking the conjugate of equation (1) and multiplying the left side by $u(r)$ we obtain

$$
-2 i k u \frac{\partial}{\partial z} u^{*}+u \nabla^{2} u^{*}=0
$$

Subtracting equation (3) from (2) gives

$$
2 i k\left(u^{*} \frac{\partial}{\partial z} u+u \frac{\partial}{\partial z} u^{*}\right)+u^{*} \nabla^{2} u-u \nabla^{2} u^{*}=0
$$

Equation (4) may be simplified as follows

$$
2 i k \frac{\partial}{\partial z}\left(u u^{*}\right)+u^{*} \nabla^{2} u-u \nabla^{2} u^{*}=0
$$

Since $u u^{*}=I$ we have 


$$
2 i k \frac{\partial}{\partial z}(I)+u^{*} \nabla^{2} u-u \nabla^{2} u^{*}=0
$$

To simplify the last equation we make use of the vector identity

$$
u^{*} \nabla^{2} u-u \nabla^{2} u^{*}=\nabla \cdot\left(u^{*} \nabla u-u \nabla u^{*}\right)
$$

thus equation (6) becomes

$$
2 i k \frac{\partial}{\partial z}(I)+\nabla \cdot S=0
$$

where $S=u^{*} \nabla u-u \nabla u^{*}$. Since $u(r)=I^{l / 2}(r) \exp (i \varphi(r))$ one obtains $u^{*} \nabla u-u \nabla u^{*}=2 i \Gamma \varphi$ and thus equation (8) becomes, after simplification,

$$
k \frac{\partial}{\partial z}(I)+\nabla \cdot(I \nabla \varphi)=0
$$

Now if we substitute the phase $\varphi$ for $k W$, where the function $W=W(x, y)$ is the aberration function, we obtain the equation for conservation of irradiance

$$
\frac{\partial}{\partial z}(I)+\nabla \cdot(I \nabla W)=0
$$

At this point we may recall that equation (10) is similar to equation for the conservation of electric charge. Performing the volume integral of equation (9) and aplying the divergence theorem $\int_{V} \nabla \cdot(I \nabla \varphi) d v=\int_{S}(\nabla \nabla) \cdot d a$ to the second term, one obtains $\int \frac{\partial I}{\partial z} d v+\int \nabla \nabla \cdot d a=0$.

Considering that $\nabla W$ and $d a$ are almost parallel and that $d v=d a d z$, the integral is satisfied only if $\partial I+I \nabla W=0$, from which we finally obtain

$$
|\nabla W|=-\frac{\partial I}{I}
$$

Equation (11) shows that the modulus of the gradient of the aberration function is equal to the term $\frac{\partial I}{I}$ which can be interpreted as a relative variation of $I$. Recording measurements of $\frac{\nabla I}{I}$ in two transverse planes along the optic axis, we thus may obtain information on the aberration function.

\section{ACKNOWLEDMENTS}

We acknowledment the support of Consejo Nacional de Ciencia y Tecnologia (CONACYT), México.

\section{REFERENCES}

1. Roddier F., "Wavefront sensing and the irradiance transport equation", Appl. Opt. 29, 1402, (1990). 